\title{
Reactive Oxygen Species Production in the Isolated Perfused Rat Liver: Effect of Respiratory Inhibitors
}

\author{
Masahiro OKuda, ${ }^{1, *}$ Hsien-Change LeE, ${ }^{1}$ Britton Chance, ${ }^{1}$ \\ and Chellappa KumAR ${ }^{2}$ \\ ${ }^{1}$ Department of Biochemistry and Biophysics, University of Pennsylvania, \\ Philadelphia, PA 19104-6089, USA \\ ${ }^{2}$ Institute for Biophysical Biomedical Research, 3401 Market Street, \\ No. 100, Philadelphia, PA 19104, USA
}

(Received June 24, 1992)

\begin{abstract}
Summary Mitochondria are one of the possible sources of potentially harmful reactive oxygen species in tissue during ischemia-reperfusion. In order to delineate the capacity of the respiratory chain to produce reactive oxygen species, the effect of respiratory inhibitors on the oxygen uptake and luminol and lucigenin enhanced chemiluminescence was measured in isolated blood-free perfused rat livers. Concomitant tissue damage was monitored by measuring the release of lactate dehydrogenase and thiobarbituric acid reactive substances by the livers. Cyanide inhibited oxygen uptake reversibly while rotenone and Antimycin A effects were not reversible. Enhanced chemiluminescence was increased immediately by cyanide, suggesting increased formation of reactive oxygen species. Antimycin A produced a delayed, large increase in chemiluminescence concurrent with severe cell damage and increased lipid peroxidation; Cyanide and rotenone induced only limited cell damage. The cell damaging effect of Antimycin A was delayed by the simultaneous administration of cyanide. These results are discussed in terms of the possible role of reactive oxygen species produced during Antimycin A inhibition of the respiratory chain in cell damage.
\end{abstract}

Key Words: oxygen radicals, perfused liver, respiratory inhibitors, chemiluminescence

The possible involvement of reactive oxygen species (ROS) in tissue damage under different pathological conditions $[1,2]$ has led to recent interest in the measurement of ROS levels in tissue. We and others have recently reported on the

* To whom correspondence should be addressed. Present address: Department of Anesthesiology, Mie University School of Medicine, Tsu 514, Japan 
successful application of enhanced chemiluminescence procedures to study ROS production in perfused organs during ischemia-reperfusion and other similar conditions [3-7].

Hepatocytes in the presence of respiratory inhibitors have been used extensively as models of 'chemical ischemia' and the consequent cell damage has been studied under different conditions. Possible involvement of ROS in tissue damage during 'chemical ischemia' has been considered [8-12]. An interesting corollary to studies of 'chemical ischemia' is whether there exists an ROS mediated chemical reperfusion injury such as has been suggested to be present during normal ischemia-reperfusion. Since respiratory inhibition of the perfused liver may be responsible upon inhibitor washout, this constitutes a good model system in which such a study may be performed and any ROS burst present upon the lifting of inhibition identified.

In another vein, the steady state production of peroxide and, possibly as a consequence, other ROS has been studied extensively in the perfused liver; it is known from these studies that peroxisomes and mitochondria produce significant amounts of peroxide [13-21]. Spin trapping studies on mitochondria have also established the production of significant levels of superoxide in the respiratory chain [20]. Mitochondrial superoxide production was enhanced by Antimycin A [17-21] and cyanide suppressed the Antimycin A stimulated superoxide production [19]. Since the liver cells normally contain significant concentrations of superoxide dismutase and catalase, it needs to be established whether the increased mitochondrial production of ROS upon blockade of the respiratory electron transport chain translates into significantly increased tissue levels of ROS. This will also have a bearing on the extent of involvement of mitochondrially generated ROS on tissue damage under pathological conditions. Given these conditions, we have studied the effect of the respiratory inhibitors cyanide, Antimycin A and rotenone singly and in combination on the enhanced chemiluminescence, cell damage and lipid peroxidation in the isolated perfused rat liver and present the results here.

\section{MATERIALS AND METHODS}

Male Sprague-Dawley rats (200-250 g body weight) were obtained from Charles River Co. (Wilmington, MA). Each rat, fasted for $24 \mathrm{~h}$ before use, was heparinized (sodium heparin, 1,000 units/kg, i.p.) and anesthetized (sodium pentobarbital, $50 \mathrm{mg} / \mathrm{kg}$, i.p.) before liver isolation. The liver isolation and perfusion were carried out according to Miller [22]. After surgery, livers were excised from abdominal cavity and transferred to the light-tight box for chemiluminescence measurements. Livers were perfused with blood-free Krebs-Henseleit

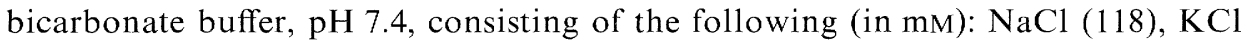
(4.7), $\mathrm{KH}_{2} \mathrm{PO}_{4}$ (1.25), $\mathrm{CaCl}_{2}$ (1.25), $\mathrm{MgSO}_{4}$ (1.25), $\mathrm{NaHCO}_{3}$ (24.0), and glucose (11.1), bubbled continuously with a gaseous mixture containing $95 \% \mathrm{O}_{2}-5 \% \mathrm{CO}_{2}$, 
and filtered through a $0.45 \mu \mathrm{m}$ Millipore filter. The inflow was through the portal vein and the outflow was through the inferior vena cava. The hepatic artery was ligated. Both the inflow and outflow vessels were cannulated and the perfusate was pumped through the liver with a peristaltic pump (Masterflex, Cole Parmar Instrument Co., Chicago, IL) at a constant flow rate of $3-3.5 \mathrm{ml} / \mathrm{min}$ per g wet weight of the liver in a nonrecirculating system. The perfusate was maintained at $36.0 \pm 0.5^{\circ} \mathrm{C}$ throughout the study using a water jacket. A clark-type oxygen electrode (Standard Oxygen Probe, Yellow Springs Instrument Co., Yellow Springs, $\mathrm{OH}$ ) was situated in-line and oxygen uptake was calculated from the influent minus effluent oxygen concentration difference, the flow rate and the liver wet weight.

Detection of chemiluminescence. The rat liver under perfusion was enclosed in a light-tight perfusion box and chemiluminescence measured by an EMI 9658 photomultiplier at an applied potential of $0.95 \mathrm{kV}$. The photomultiplier was cooled to $-20^{\circ} \mathrm{C}$ to decrease the dark count. Standard high performance single photon counting electronics consisting of a low noise preamplifier, amplifier, discriminator and ratemeter were used for continuous measurement of the tissue chemiluminescence. Chemiluminescence data are expressed as count per second (cps). In order to compare the chemiluminescence intensities from different livers, photoemission intensities were normalized to unit surface area of the liver (cps/ $\left.\mathrm{cm}^{2}\right)$.

Experimental protocol. All livers were perfused for $10 \mathrm{~min}$ with the standard K-H perfusate. Subsequently perfusate containing two different chemiluminescence enhancers $(0.2 \mu \mathrm{M}$ luminol and $0.01 \mu \mathrm{M}$ lucigenin $)$ was begun to administer through the rest of the study. Afterward, livers were randomly divided for each inhibitor experiments.

Each inhibitor was administered at the chosen concentration for $30 \mathrm{~min}$. Some livers were infused with cyanide for $90 \mathrm{~min}$. The inhibitor solutions were infused into the perfusion line close to the point of entry into the liver at a concentration sufficient to produce the desired final concentrations in the perfusion buffer. The final concentrations of the inhibitors in the perfusion buffer were $0.15 \mathrm{mM}, 10 \mu \mathrm{g}$ / $\mathrm{ml}$ and $0.5 \mu \mathrm{M}$ for potassium cyanide, Antimycin A and rotenone, respectively.

Release of $L D H$ and TBARS. LDH activity in effusate was determined using Sigma diagnostic kit DG 1340-KI (Sigma, St. Louis, MO). TBARS was measured using a modified thiobarbituric acid fluorometric method [23]. Briefly, $1 \mathrm{ml}$ of effusate was mixed thoroughly with $2 \mathrm{ml}$ of a reagent containing $15 \%$ trichloroacetic acid, $0.375 \%$ thiobarbituric acid, and $0.25 \mathrm{~N}$ hydrochloric acid and was heated for $20 \mathrm{~min}$ at $95^{\circ} \mathrm{C}$ in the test tube heater; after cooling, samples were centrifuged at $1,000 \times g$ for $10 \mathrm{~min}$ and the fluorescence of the supernatant was determined at $515 \mathrm{~nm}$ excitation and $553 \mathrm{~nm}$ emission with a Luminescence Spectrometer (Model LS-30, Perkin Elmer, Buckinghamshire, England). This modification did not show any effect on the TBARS value in the effusate obtained under our experimental conditions. Concentration of TBARS in the effusate was 
obtained by comparison with malondialdehyde standards (1,1,3,3-tetraethoxypropane) and the TBARS release rate was determined using the flow rate and the liver wet weight.

Chemicals used in this study were purchased from Sigma Chemical Co. (St. Louis, MO).

Statistics. All data are expressed as mean \pm SD. Statistical analysis was performed using analysis of variance followed by Fisher protected least significant difference test. $p<0.05$ was considered statistically significant.

\section{RESULTS}

Figure 1 shows the effect of the different respiratory inhibitors on oxygen uptake by the liver. Cyanide administration leads to about 50\% inhibition of oxygen uptake compared to the control values; upon termination of cyanide infusion, the oxygen uptake returns to control values over about $10 \mathrm{~min}$; thus, the inhibition of respiration by cyanide seems to be completely reversible. Sustained

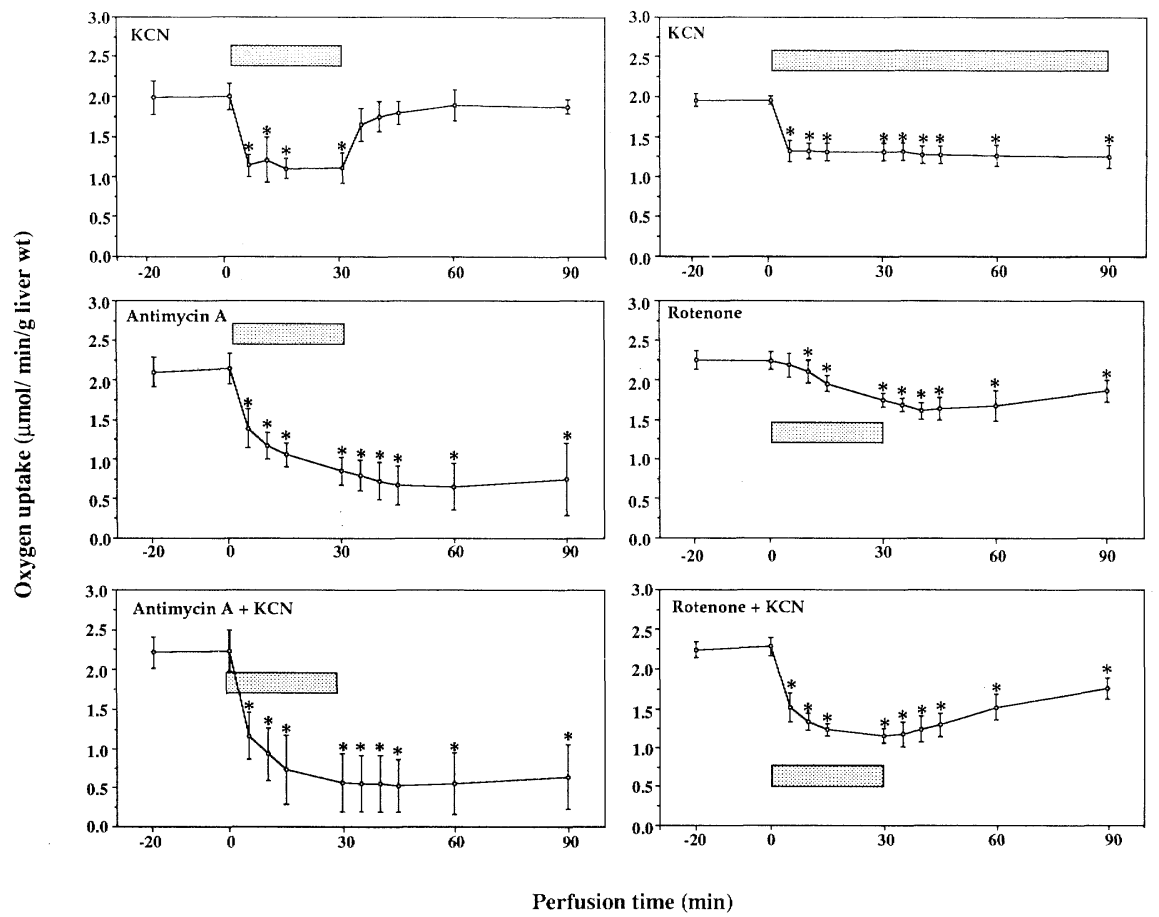

Fig. 1. Effect of respiratory inhibitors on oxygen uptake by Krebs-Henseleit buffer perfused isolated rat livers at $36 \pm 0.5^{\circ} \mathrm{C}$ : the inhibitors were included in the perfusion buffer for the duration indicated by the bars ( $\square$ ). The concentrations of inhibitors are described in the text. Values are mean $\pm \mathrm{SD}$ of $n=5$ experiments per group. ${ }^{*} p<0.05$ compared with the values prior to inhibitors infusion. 
cyanide administration, as shown in the second panel, leads to sustained and steady inhibition of oxygen uptake. At the concentrations used, Antimycin A inhibits oxygen uptake in the liver by about $75 \%$ and rotenone by about $35 \%$ [24]; the effects of these two inhibitors are not reversible in that there is no recovery of oxygen uptake upon termination of the administration of the inhibitors. When Antimycin A+cyanide are administered, there is no significant increase in the extent of inhibition over that seen with Antimycin A alone and, consistent with the results obtained from the infusion of Antimycin A alone, there is no recovery of oxygen uptake when the administration of the inhibitors is terminated. When rotenone + cyanide are administered, the extent of inhibition is increased over that seen with the administration of rotenone alone; on termination of inhibitor administration, oxygen uptake partially recovers to near values seen with administration of rotenone alone. This is consistent with cyanide component of the inhibition of oxygen uptake being reversible.

Figure 2 shows the time course of luminol enhanced chemiluminescence (Lm-CL) of the liver under perfusion in the presence of the different inhibitors.
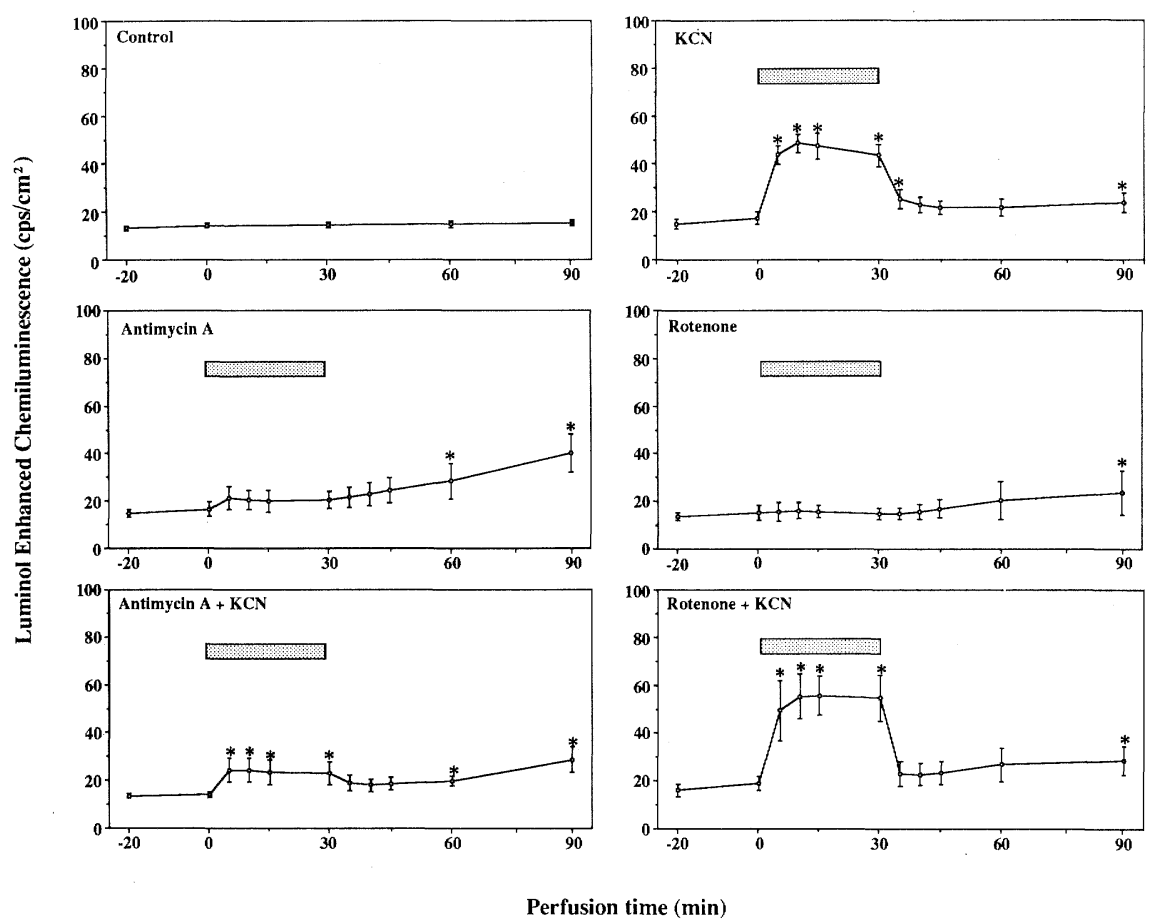

Fig. 2. Effects of respiratory inhibitors on the luminol enhanced chemiluminescence (Lm$\mathrm{CL}$ ) of isolated perfused rat livers; conditions as in Fig. 1. The inhibitors were included in the perfusion buffer for the duration indicated by the bars ( $\square$ ). Values are mean \pm SD of $n=5$ experiments per group. ${ }^{*} p<0.05$ compared with the values prior to inhibitors infusion. cps, count per second. 
The control livers show small increases in Lm-CL over $90 \mathrm{~min}$ of perfusion. Cyanide administration leads to an immediate increase in the Lm-CL intensity to about 2.5 times the control value. Termination of cyanide administration, consistent with the oxygen uptake results, leads to a return of the chemiluminescence to near control values over the next $10 \mathrm{~min}$. Antimycin A leads to only very small increases in Lm-CL on administration. However, Antimycin A leads to a continuous increase in Lm-CL after termination of administration, resulting in significantly elevated chemiluminescence compared to controls. Antimycin A+cyanide leads to small but significant increases in Lm-CL immediately on administration. Antimycin A and cyanide are mutually antagonistic in their effects on the Lm-CL of the liver: the increase in $\mathrm{Lm}-\mathrm{CL}$ seen during cyanide administration is largely blocked by simultaneous administration of Antimycin A; the increase in Lm-CL seen at longer times during administration of Antimycin A alone is delayed by the simultaneous administration of cyanide for a period. Rotenone alone has only minor effect on the Lm-CL of the liver; effect of rotenone + cyanide administration on Lm-CL seems to be dominated by the effect of cyanide.

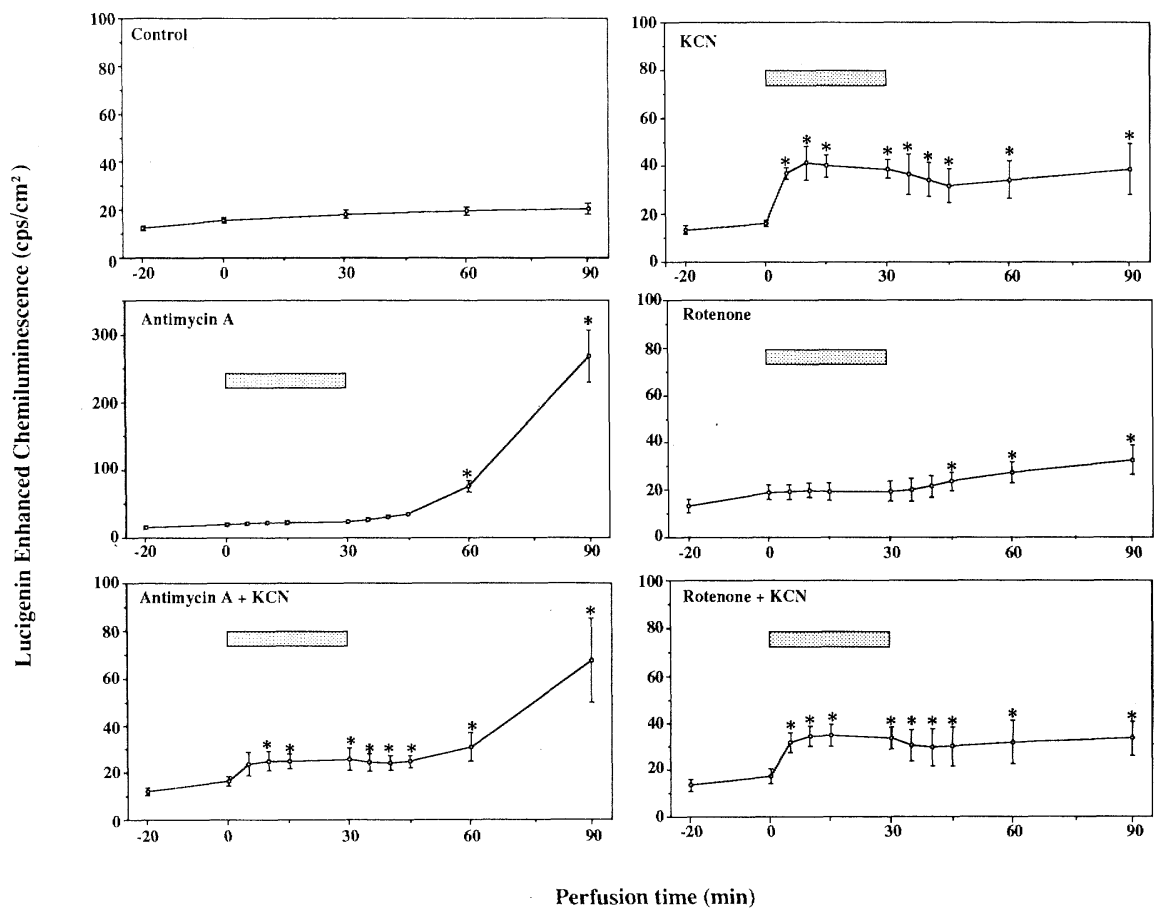

Fig. 3. Effects of respiratory inhibitors on the lucigenin enhanced chemiluminescence (Lg-CL) of isolated perfused rat livers; conditions as in Fig. 1. The inhibitors were included in the perfusion buffer for the duration indicated by the bars ( $\square$ ). Values are mean \pm SD of $n=5$ experiments per group. ${ }^{*} p<0.05$ compared with the values prior to inhibitors infusion. cps, count per second. 
Figure 3 shows the time course of lucigenin enhanced chemiluminescence ( $\mathrm{Lg}-\mathrm{CL}$ ) during administration of the same inhibitors. The control livers show a slightly larger increase in Lg-CL in the course of the perfusion compared studies in Lm-CL. Cyanide administration leads to an immediate increase in the chemiluminescence intensity, as is the case with Lm-CL; however, on termination of cyanide administration, unlike Lm-CL, Lg-CL does not return to the control values, but remains slightly elevated. Antimycin A behaves similar to the Lm-CL studies; there is no immediate increase in the Lg-CL intensity, but, after about 4050 min of perfusion, the Lg-CL continuously increases, to about 10 times higher than control in about 90 min (note the different scale used for the Antimycin A data). Again, cyanide, and Antimycin A are mutually antagonistic in their effect on Lg-CL; the simultaneous administration of cyanide and Antimycin A leads to lower immediate increases in $\mathrm{Lg}-\mathrm{CL}$ than administration of cyanide alone and Lg-CL increases at later times are lower than that produced by Antimycin A alone. Rotenone has only a minor effect on the Lg-CL time course while the effects of

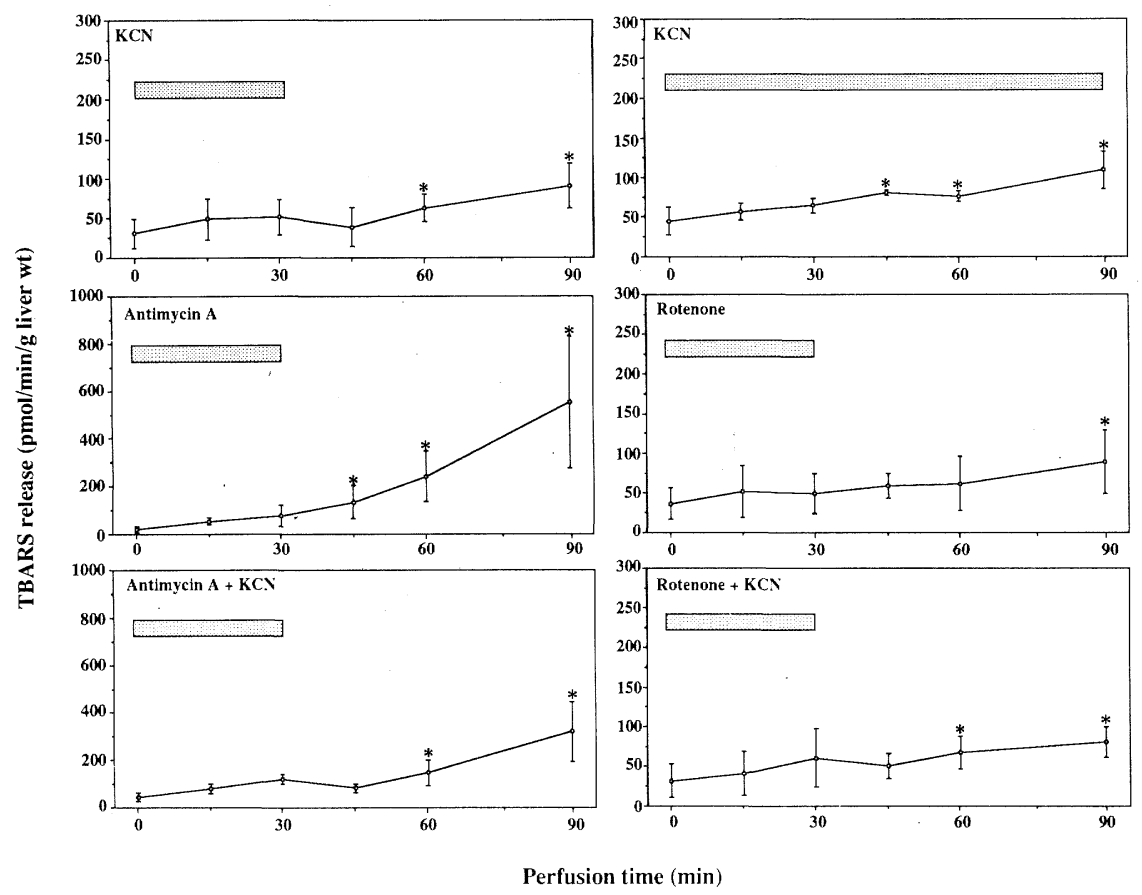

Fig. 4. Effects of respiratory inhibitors on the release of TBARS into the perfusate by isolated perfused rat livers; conditions as in Fig. 1. Livers of each group were perfused with standard $\mathrm{K}-\mathrm{H}$ buffer for a period of $30 \mathrm{~min}$ before the administration of inhibitors. The inhibitors were included in the perfusion buffer for the duration indicated by the bars ( with the values prior to inhibitors infusion. TBARS, thiobarbituric acid reactive substances. 
rotenone + cyanide are dominated by the effect of cyanide administration.

Figure 4 shows the effect of the inhibitors on the release of TBARS into the perfusate: in control livers (not shown) the level of TBARS in the perfusate did not change significantly in the course of $120 \mathrm{~min}$ of perfusion (include $30 \mathrm{~min}$ equilibration). No larger effects were seen on the time course of TBARS release due to administration of cyanide, rotenone or rotenone + cyanide. Antimycin A administration, however, leads to very large increases at later times (note the different scale used for the Antimycin A data). Simultaneous administration of Antimycin A and cyanide, as with the chemiluminescence studies, leads to increases lower than induced by Antimycin A alone and higher than induced by cyanide.

Figure 5 presents the time course of $\mathrm{LDH}$ release from the perfused liver: in control livers, $\mathrm{LDH}$ release increased slowly from 10 to $30 \mathrm{mU} / \mathrm{min} / \mathrm{g}$ liver wt in the course of perfusion (not shown). Cyanide, administered for either $30 \mathrm{~min}$ or 90 min led to significant increases in the LDH release; rotenone and rotenone + cyanide also behaved similarly. Antimycin A treated livers, however, showed very large increases in LDH release, about 100 times higher than the control livers.

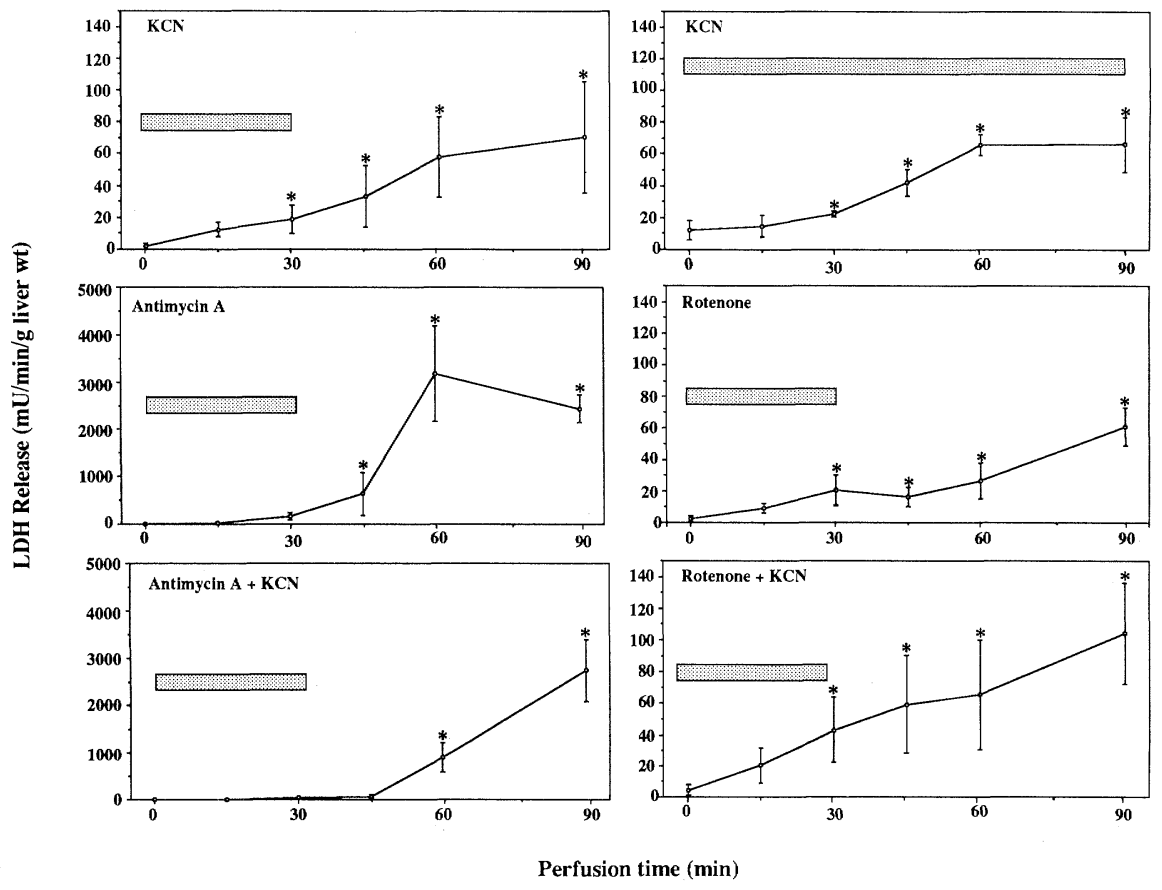

Fig. 5. Effects of respiratory inhibitors on the release of LDH into the perfusate by isolated perfused rat livers; conditions as in Fig. 1. Livers of each group were perfused with standard $\mathrm{K}-\mathrm{H}$ buffer for a period of $30 \mathrm{~min}$ before the administration of inhibitors. The inhibitors were included in the perfusion buffer for the duration indicated by the bars (ख). Values are mean $\pm \mathrm{SD}$ of $n=5$ experiments per group. ${ }^{*} p<0.05$ compared with the values prior to inhibitors infusion. LDH, lactate dehydrogenase. 
Administration of cyanide for 30 min, simultaneous with Antimycin A, delayed the Antimycin A induced increase in LDH release.

In separate experiments (not shown), we studied the effect of cyanide administration on the $\mathrm{Lg}$-CL of livers isolated from 3 -amino-1,2,4-triazole $(1 \mathrm{~g} / \mathrm{kg}$ body weight; i.p.) treated rats [25]. No significant differences were seen in the effect of cyanide at the concentrations used on the chemiluminescence behavior of livers isolated from normal and amino triazole treated rats.

In other experiments, we perfused the liver with higher concentration of cyanide $(1.5 \mathrm{~mm} / \mathrm{ml})$ : this led to $80-85 \%$ inhibition of oxygen uptake by the organ and massive enzyme release after about $60 \mathrm{~min}$ of cyanide administration, similar to that seen on application of Antimycin A. TBARS release was also similar enhanced on high cyanide administration.

\section{DISCUSSION}

Lm-CL and Lg-CL are immediately increased by cyanide but not Antimycin A; The effect of cyanide is partly inhibited by Antimycin $A$

Previous studies, especially on mitochondria or submitochondrial particles, suggest that ROS formation is increased by Antimycin A [17-21] and that cyanide inhibits such Antimycin A induced increases in ROS production [19]. This has been rationalized based on the interpretation that superoxide is formed from ubisemiquinone upstream of the Antimycin A binding site and that cyanide decreases Antimycin A induced ROS production by inhibiting the oxidation of the reduced quinone and thus preventing the formation of the semiquinone upstream of the Antimycin A binding site. Our present results are exactly the opposite: Cyanide increases $\mathrm{Lm}-\mathrm{CL}$ and $\mathrm{Lg}-\mathrm{CL}$ suggesting increased tissue ROS levels and Antimycin A inhibits such cyanide induced increases in ROS. Our present results thus suggest that under our experimental conditions ROS production in the mitochondrial respiratory chain occurs at a site downstream of the Antimycin A binding site and upstream of the cyanide binding site, i.e., between Cytochrome $b-562$ and Cytochrome oxidase. In this stretch of the respiratory chain, the most likely candidates for the source of ROS are the Antimycin A sensitive or TTFA sensitive ubisemiquinone radical and, possibly, fully reduced iron sulfur proteins. That Antimycin A does not increase ROS production as reported by chemiluminescence study and this indicates that in the isolated perfused liver, under our conditions, respiratory chain ROS production occurs in the stretch downstream of Antimycin A binding site. Indeed, peroxide production from the site upstream of Antimycin A was found to be significantly modulated by several variables including the presence of uncouplers, the nature of the substrate etc. [19]. Thus, it is possible that, under the conditions of the present experiments, the ROS production site upstream of the Antimycin A binding site is not a significant source of ROS while a site downstream of the Antimycin A binding site is.

Mitochondria are not the only source of ROS in the liver: significant peroxide

Vol. 15, No. 2, 1993 
formation was found in the peroxisomes and microsomes as well using subcellular preparations [2]. Peroxide production from microsomes and peroxisomes was found to be significantly stimulated by the addition of appropriate substrates [13]. It is conceivable that these non-mitochondrial sources contribute significantly to the steady state ROS levels in the perfused liver; and that the effect of cyanide is to increase the reduction level of pyridine nucleotides (or other similar substrates) which then serve as substrates for the microsomal production of ROS. However, if this were the case, Antimycin A also would be expected to lead to increased ROS production since Antimycin A also would lead to increased reduction of pyridine nucleotides. Our results show that Antimycin A does not lead to higher steady state levels of ROS in the perfused liver as monitored by $\mathrm{Lm}-\mathrm{CL}$ and $\mathrm{Lg}-\mathrm{CL}$. Further, Antimycin A inhibits the effects of cyanide on the Lm-CL and LG-CL, suggesting that the effect of cyanide can not be explained as being due to increased reduction of the pyridine nucleotide pool.

Another possible explanation for the effect of cyanide is that cyanide increases the steady state levels of ROS in the liver not only by enhancing production of ROS but also by inhibiting ROS metabolizing enzymes [12, 13]. To test this possibility with reference to catalase, we compared the effect of cyanide on 3-amino-1,2,4-triazole treated and normal rat liver: no significant differences were seen in Lg-CL in the control or cyanide treated conditions between the two sets of livers, suggesting minimal contribution of catalase inhibition to the effects of cyanide. Further, Antimycin A will not be expected to inhibit the effect of cyanide if the major site of action of cyanide was not the respiratory chain. Thus, the most likely explanation for the effect of cyanide and Antimycin A on the Lm-CL and $\mathrm{Lg}-\mathrm{CL}$ of the liver is the presence of a site of ROS production on the respiratory chain between the Antimycin A and cyanide sites.

Rotenone, while slowly inhibiting oxygen uptake to a limited extent, does not lead to any significant effects on Lm-CL and Lg-CL. This suggests that partial inhibition of the NADH linked substrate oxidation does not result in increased ROS production. The effects of rotenone + cyanide are very close to that of cyanide alone suggesting that sufficient electron flow from the succinate side of the respiratory chain is available so as to limit any possible effect of rotenone in the system.

There are significant differences between the time courses of $\mathrm{Lm}$-CL and $\mathrm{Lg}-\mathrm{CL}$ changes

This clearly evident, for example, when the effect of termination of cyanide administration is examined. While $\mathrm{Lm}-\mathrm{CL}$ rapidly returns to near control values, Lg-CL remains significantly elevated after termination of cyanide infusion. Lm$\mathrm{CL}$ is rather nonselective with respect to the chemical nature of the ROS and is enhanced in the presence of heme + peroxide and other similar oxidants [26]. $\mathrm{Lg}-\mathrm{CL}$ is considered to be more selective and indicative of the presence of superoxide [27]. Hence, some of the differences in the behavior of Lm-CL and Lg-CL may be a consequence of such differences in chemical sensitivity between luminol and 
lucigenin and reflect changes in the composition of ROS in the liver at different times. Yet another possible source of differences between Lm-CL and Lg-CL arises from the observation that lucigenin is taken up by hepatocytes [28]. We find that $\mathrm{Lm}-\mathrm{CL}$ in the liver is more than $80 \%$ inhibited by the infusion of superoxide dismutase (SOD) while Lg-CL is only about $10-20 \%$ sensitive to SOD (unpublished observation). In in vitro, both $\mathrm{Lm}-\mathrm{CL}$ and $\mathrm{Lg}-\mathrm{CL}$ are almost completely abolished by the inclusion of SOD in model chemical systems [7]. The differences in sensitivity of $\mathrm{Lm}-\mathrm{CL}$ and $\mathrm{Lg}-\mathrm{CL}$ to added SOD in the perfused liver are caused by different accessibilities of luminol and lucigenin to the enzyme, suggesting that a large part of $\mathrm{Lm}-\mathrm{CL}$ arises from within the vascular compartment, while a large component of $\mathrm{Lg}-\mathrm{CL}$ arises from the intracellular compartment. These differences, if significant, would suggest that, as reflected by $\mathrm{Lg}-\mathrm{CL}$, mitochondrial ROS production continues to be significantly elevated and does not return to the control levels on termination of cyanide administration. Similar increase in Lg-CL are seen in the case of rotenone or rotenone +cyanide administration as well.

\section{Antimycin $A$ leads to delayed, large increases in Lg-CL, enzyme release and thiobarbituric acid reactive substances}

Antimycin A administration leads to extensive cell damage as evidenced by large release of $\mathrm{LDH}$ into the perfusate beginning at about $50 \mathrm{~min}$ after the start of Antimycin A administration. The LDH release is simultaneous with the release of lipid peroxidation products into the perfusate as well as very large increase in $\mathrm{Lg}-\mathrm{CL}$. Since the large increase in $\mathrm{Lg}-\mathrm{CL}$ does not precede the enzyme release significantly, we consider the enhanced ROS production, as indicated by the enhanced Lg-CL, to result from cell damage rather than to be the cause of cell damage. That on disruption of tissue the chemiluminescence levels increase several fold is well established. Thus, we see the massive enzyme release, lipid peroxidation and $\mathrm{Lg}-\mathrm{CL}$ increases all to be simultaneous indicators of some tissue damage process brought about by the administration of Antimycin A. Cyanide and rotenone also lead to significant increases in enzyme release compared to control livers, but these increases are much smaller than that induced by Antimycin A. Release of TBARS also is increased by cyanide, rotenone or rotenone + cyanide but to a much larger extent by Antimycin A. At the concentration used in the current studies, prolonged $(90 \mathrm{~min})$ application of cyanide does not lead to significantly more enzyme release or TBARS release than does short $(30 \mathrm{~min})$ application suggesting that the $2-3$ fold increases seen in these parameters is mainly associated with the effects of prolonged perfusion on the liver rather than the specific effects of these inhibitors. In separate experiments we perfused livers with 10 fold higher concentrations of cyanide; consistent with previous studies [11], these livers showed about $85 \%$ inhibition of oxygen uptake and massive enzyme release and TBARS release comparable to those seen with Antimycin A in the current study beginning about 15 min after the initiation of cyanide infusion. Thus, both high concentrations of cyanide and Antimycin A lead to significant 
ischemic injury.

There is no correlation between the early $L m-C L$ or $L g-C L$ response and cell damage

Cyanide increases $\mathrm{Lm}-\mathrm{CL}$ and $\mathrm{Lg}-\mathrm{CL}$ significantly on application as shown during the present study, but does not lead to significant tissue damage at the concentrations used. Antimycin A does not give rise to any immediate increases in Lm-CL or Lg-CL, but eventually leads to significant cell damage, as revealed by large increases in enzyme and TBARS release as well as in the delayed Lg-CL increase. Thus, tissue damage in these cases would seem to be a consequence of energy depletion and not correlated with the tissue ROS levels. It is still possible that ROS concentrations in some specific tissue compartments are involved in tissue damage, but the current study is designed to explore that possibility. An alternate, though trivial, interpretation will be that neither $\mathrm{Lm}-\mathrm{CL}$ nor Lg-CL are good measures of tissue ROS levels; while calibration of Lm-CL or Lg-CL in terms of absolute tissue ROS levels is not as yet possible, mainly due to problems associated with corrections for the absorption of the chemiluminescent photons by the tissue, in relative terms correlation of $\mathrm{Lm}-\mathrm{CL}$ and $\mathrm{Lg}-\mathrm{CL}$ with tissue ROS levels is likely to be valid. Thus, tissue ROS would not seem to play a significant role in cell injury related to respiratory inhibition. This, however, does not rule out the possibility that other chemicals (such as the ubisemiquinone radical) may be present at higher than normal steady state levels in mitochondria in presence of respiratory inhibitors, with associated toxic consequences for the cell [20].

\section{Low concentrations of cyanide delays the onset of Antimycin $A$ induced cell damage}

All indicators of Antimycin A induced cell damage measured here, LDH release, TBARS release, large increases in Lg-CL showed that the time course of tissue damage was significantly slowed by the simultaneous presence of low concentrations of cyanide. If energy deprivation is the simple cause of tissue damage, as one may conclude from the observation that both Antimycin A as well as higher concentrations of cyanide lead to significant cell damage, it would be expected that the presence of cyanide will lead to either an increase or have no effect on Antimycin A induced cell damage. Our finding that cyanide delays the effect of Antimycin A on the liver suggests a more direct and specific interaction between the effects of cyanide and Antimycin A. In the heart, reperfusion induced enzyme release has been shown to be inhibited by the presence of cyanide; this was interpreted to mean that enzyme release was an energy dependent phenomenon [29]. In the present case such an explanation is not applicable since liver respiration is inhibited by the Antimycin A even after the cyanide is withdrawn. Thus the effect of cyanide on cell damage has to be ascribed to some other effect of cyanide. Given that cyanide at high concentrations can by itself give rise to large enzyme release from the liver, it is not likely that cyanide directly inhibits the enzyme 
release process i.e. bleb formation or release. Cyanide is known to inhibit the Antimycin A induced increase in the steady state concentration of ubisemiquinone and, consequently, the production of superoxide in mitochondria [20]. This effect may be relevant in explaining the effect of cyanide in delaying the Antimycin A induced cell damage.

To summarize, we find that ROS levels in the isolated perfused liver are increased by low concentrations of cyanide; significant cell damage is caused by Antimycin A; Antimycin A and cyanide are mutually inhibitory with respect to tissue properties. Clearly, further work is necessary to delineate the dynamics of this interrelationship in the liver.

Research supported in part by NIH grants AA 07186 and HL 18708.

\section{REFERENCES}

1. Piper, H.M. (ed.) (1990): Pathophysiology of Severe Ischemic Injury, Kluwer Academic Publishers, Dordrecht.

2. Halliwell, B., and Gutteridge, J.M.C. (1989): Free Radicals in Biology and Medicine, Clarendon Press, Oxford.

3. Kumar, C., Okuda, M., Ikai, I., and Chance, B. (1990): Luminol enhanced chemiluminescence of the perfused rat heart during ischemia and reperfusion. FEBS Lett., 272, 121-124.

4. Okuda, M., Ikai, I., Chance, B., and Kumar, C. (1991): Superoxide dismutase, but not catalase or mannitol, decreased oxygen radical formation in perfused rat heart during ischemia-reperfusion as monitored by luminol enhanced chemiluminescence. J. Appl. Cardiol., 6, 113-121.

5. Okuda, M., Ikai, I., Chance, B., and Kumar, C. (1991): Oxygen radical production during ischemia-reperfusion in the isolated perfused rat liver as monitored by luminol enhanced chemiluminescence. Biochem. Biophys. Res. Commun., 174, 217-221.

6. Henry, T.D., Archer, S.L., Nelson, D., Weir, E.K., and From, A.H.L. (1990): Enhanced chemiluminescence as a measure of oxygen-derived free radical generation during ischemia and reperfusion. Circ. Res., 67, 1453-1461.

7. Archer, S.L., Nelson, D.P., and Weir, E.K. (1989): Detection of activated $\mathrm{O}_{2}$ species in vitro and in rat lungs by chemiluminescence. J. Appl. Physiol., 67, 1912-1921.

8. Gores, G.J., Nieminen, A., Fleishman, K.E., Dawson, T.L., Herman, B., and Lemasters, J.J. (1988): Extracellular acidosis delays the onset of cell death in ATP-depleted hepatocytes. Am. J. Physiol., 255, C315-C322.

9. Gores, G.J., Flarsheim, C.E., Dawson, T.L., Nieminen, A., Herman, B., and Lemasters, J.J. (1989): Swelling, reductive stress, and cell death during chemical hypoxia in hepatocytes. Am. J. Physiol, 257, C347-C354.

10. Aw, T.Y., and Jones, D.P. (1989): Cyanide toxicity in hepatocytes under aerobic and anaerobic conditions. Am. J. Physiol., 257, C435-C441.

11. Younes, M., and Strubelt, O. (1990): Protection by exogenous glutathione against hypoxic and cyanide-induced damage to isolated perfused rat livers. Toxicol. Lett., 50, 229-236.

12. Jaeschke, H., and Mitchell, J.R. (1989): Mitochondria and xanthine oxidase both generate reactive oxygen species in isolated perfused rat liver after hypoxic injury. Biochem. Biophys. Res. Commun., 160, 140-147.

13. Chance, B., and Oshino, N. (1971): Kinetics and mechanism of catalase in peroxisomes of the mitochondrial fraction. Biochem. J., 122, 225-233.

14. Sies, H., and Chance, B. (1970): The steady state level of catalase compound I in isolated hemoglobin-free perfused rat liver. FEBS Lett., 11, 172-176.

Vol. 15, No. 2, 1993 
15. Oshino, N., Chance, B., Sies, H., and Bucher, T. (1973): The role of $\mathrm{H}_{2} \mathrm{O}_{2}$ generation in perfused rat liver and the reaction of catalase compound I and hydrogen donors. Arch. Biochem. Biophys., 154, 117-131.

16. Oshino, N., and Chance, B. (1975): The properties of sulfite oxidation in perfused rat liver; interaction of sulfite oxidase with the mitochondrial respiratory chain. Arch. Biochem. Biophys., 170, 514-528.

17. Loschen, G., Flohe, L., and Chance, B. (1971): Respiratory chain linked $\mathrm{H}_{2} \mathrm{O}_{2}$ production in pigeon heart mitochondria. FEBS Lett., 18, 261-264.

18. Boveris, A., Oshino, N., and Chance, B. (1972): The cellular production of hydrogen peroxide. Biochem. J., 128, 617-630.

19. Boveris, A., and Chance, B. (1973): The mitochondrial generation of hydrogen peroxide. General properties and effect of hyperbaric oxygen. Biochem. J., 134, 707-716.

20. Ksenzenko, M., Konstantinov, A.A., Khomutov, G.B., Tikhonov, A.N., and Ruuge, E.K. (1983): Effect of electron transfer inhibitors on superoxide generation in the cytochrome $b c_{1}$ site of the mitochondrial respiratory chain. FEBS Lett., 155, 19-23.

21. Cadenas, E., and Boveris, A. (1980): Enhancement of hydrogen peroxide formation by protophores and ionophores in antimycin-supplemented mitochondria. Biochem. J., 188, $31-37$.

22. Miller, L.L. (1973): Technique of isolated rat liver perfusion, in Isolated Liver Perfusion and Its Application, ed. by Bartosek, I., Guaitani, A., and Miller, L.L., Raven Press, New York, pp. 11-52.

23. Yagi, K. (1976): A simple fluorometric assay for lipoperoxide in blood plasma. Biochem. Med., 15, 212-216.

24. Bucher, Th., and Sies, H. (1969) Inhibitors Tools in Cell Research, Springer-Verlag, Berlin-Heidelberg.

25. Oshino, N., and Chance, B. (1977): Properties of glutathione release observed during reduction of organic hydroperoxide, demethylation of aminopyrine and oxidation of some substances in perfused rat liver and their implications for the physiological function of catalase. Biochem. J., 162, 509-525.

26. Roswell, D.F., and White, E.N. (1978): The chemiluminescence of luminol and related hydrazides. Methods Enzymol., 57, 409-423.

27. Muller-Peddinghaus, R., and Wurl, M. (1987): The amplified chemiluminescence test to characterized antirheumatic drugs as oxygen radical scavengers. Biochem. Pharmacol., 36, $1125-1132$.

28. Hildebrandt, A.G., Weimann, A., and Kahl, R. (1986): Use of the chemiluminogenic probes luminol and lucigenin for the detection of active oxygen species in hepatic microsomes and in intact hepatocytes. Adv. Exp. Med. Biol., 197, 971-980.

29. Kehrer, J.P., Park, Y., and Sies, H. (1988): Energy dependence of enzyme release from hypoxic isolated perfused rat heart tissue. J. Appl. Physiol., 65, 1855-1860. 